\title{
A!
}

This is an electronic reprint of the original article.

This reprint may differ from the original in pagination and typographic detail.

Williamson, John; Oulasvirta, Antti; Kristensson, Per Ola

\section{Bayesian methods in interaction design (tutorial)}

DOI:

$10.1145 / 3379336.3379354$

Published: 17/03/2020

Document Version

Publisher's PDF, also known as Version of record

Please cite the original version:

Williamson, J., Oulasvirta, A., \& Kristensson, P. O. (2020). Bayesian methods in interaction design (tutorial). 1112. Paper presented at International Conference on Intelligent User Interfaces, Cagliari, Italy. https://doi.org/10.1145/3379336.3379354

This material is protected by copyright and other intellectual property rights, and duplication or sale of all or part of any of the repository collections is not permitted, except that material may be duplicated by you for your research use or educational purposes in electronic or print form. You must obtain permission for any other use. Electronic or print copies may not be offered, whether for sale or otherwise to anyone who is not an authorised user. 


\section{Bayesian Methods in Interaction Design (Tutorial)}

\author{
John Williamson \\ JohnH.Williamson@glasgow.ac.uk \\ www.dcs.gla.ac.uk/ jhw \\ University of Glasgow \\ Glasgow, United Kingdom
}

\author{
Antti Oulasvirta \\ antti.oulasvirta@aalto.fi \\ users.comnet.aalto.fi/oulasvir \\ Aalto University \\ Helsinki, Finland
}

\author{
Per Ola Kristensson \\ pok21@cam.ac.uk \\ pokristensson.com \\ University of Cambridge \\ Cambridge, United Kingdom
}

\begin{abstract}
This tutorial introduces Bayesian computational approaches to interaction and design. Bayesian methods offer a powerful approach for interactive settings with uncertainty and noise. This course introduces the theory and practice of computational Bayesian interaction, covering inference of user data and design/adaptation of interface features based around probabilistic inference. The tutorial is built around hands-on Python programming with modern computational tools, interleaved with theory and practical examples grounded in problems of wide interest in human-computer interaction.
\end{abstract}

\section{CCS CONCEPTS}

- Human-centered computing $\rightarrow$ HCI theory, concepts and models; User interface programming.

\section{KEYWORDS}

Bayesian, probabilistic, interaction design, filtering

\section{ACM Reference Format:}

John Williamson, Antti Oulasvirta, and Per Ola Kristensson. 2020. Bayesian Methods in Interaction Design (Tutorial). In 25th International Conference on Intelligent User Interfaces Companion (IUI '20 Companion), March 17-20, 2020, Cagliari, Italy. ACM, New York, NY, USA, 2 pages. https://doi.org/10. $1145 / 3379336.3379354$

\section{TOPIC}

This tutorial will cover the theory and applications of Bayesian methodology in human-computer interaction. It will cover how to model key problems in interaction design from a probabilistic perspective, and discuss practical algorithms to implement Bayesian methods in user interfaces.

The tutorial will specifically focus on two topics:

- Bayesian Optimisation to optimise parameters of an interface design efficiently from noisy, infrequent observations of user preference. Bayesian optimisation, using powerful Gaussian Process proxy functions, can elegantly trade off exploration and exploitation. It provides a consistent algorithmic methodology for human-in-the-loop optimisation of interfaces that maintains uncertainty and acknowledges the noisy nature of human responses. The tutorial will explain

Permission to make digital or hard copies of part or all of this work for personal or classroom use is granted without fee provided that copies are not made or distributed for profit or commercial advantage and that copies bear this notice and the full citation on the first page. Copyrights for third-party components of this work must be honored

For all other uses, contact the owner/author(s).

IUI '20 Companion, March 17-20, 2020, Cagliari, Italy

(c) 2020 Copyright held by the owner/author(s).

ACM ISBN 978-1-4503-7513-9/20/03.

https://doi.org/10.1145/3379336.3379354 how to represent design problems as objective functions, how to use Bayesian optimisation to perform simultaneous optimisation and acquisition and how to bring these ideas to bear on practical problems of interface design.

- Bayesian filtering can infer intention from noisy sensed observations. We can frame the interaction problem as one of recovering intention from sensors providing an incomplete and corrupted representations from a user's mind in an ongoing process. Bayesian filtering, in both the continuous space (e.g. gesture recognition) and discrete state cases (e.g. text entry), provides a consistent, efficient way to estimate intention online and construct robust interfaces. The tutorial will discuss the principles of probabilistic filtering in a user interface context, and key algorithms for Bayesian approaches, including sequential Monte Carlo and beam search.

\subsection{Relevance to IUI}

This tutorial is directly relevant to those building intelligent user interfaces. Many existing approaches in the IUI community have applied traditional machine learning and classical optimisation to improve interaction design and the Bayesian approach has received less attention. Computational advances, the recent availability of free, high-quality software libraries and a growing body of work on the theory of Bayesian inference as well as applications in interaction design are changing this. This tutorial will bring together modern approaches to implementing probabilistic, Bayesian approaches with the conceptual foundations for rethinking problems in interaction.

\section{ORGANIZERS}

- John Williamson [JHW] (primary contact) is a Lecturer in the School of Computing Science, University of Glasgow. He has a background in Bayesian filtering in mobile and touch sensing and in the representation of uncertainty in interactive systems.

- Antti Oulasvirta [AO] is an Associate Professor in the Department of Communications and Networking, Aalto University. He is an expert in optimisation approaches to interaction design and computational modeling of human behaviour.

- Per Ola Kristensson [POK] is a Professor in the Department of Engineering, University of Cambridge. He has an extensive research track record in probabilistic approaches to text entry and design of systems that incorporate uncertainty.

All organizers will attend and present as part of the tutorial. 


\section{HISTORY OF RELATED TUTORIALS}

This tutorial will present a mixture of new and revised material from prior tutorials. All of the presenters have extensive track records in successfully delivering tutorial material on computational approaches to interaction design and have refined the materials over a number of years. This event will build on an established series of full-day tutorials given at ACM SIGCHI, and from the annual Summer School on Computational Interaction. The material for this tutorial draws particularly on the tutorial on Bayesian Interaction given at SIGCHI 2019. The relevant prior events are as follows:

- 2015 Summer School on Computational Interaction, University of Glasgow, (JHW, AO, POK)

- 2016 Summer School on Computational Interaction, Aalto University, (JHW, AO, POK)

- 2017 ACM SIGCHI Tutorial on Computational Interaction, Denver, Colorado, (JHW, AO, POK)

- 2017 Summer School on Computational Interaction, ETH Zurich, (JHW, AO)

- 2018 ACM SIGCHI Tutorial on Computational Interaction, Montreal, Quebec, Canada, (AO, POK)

- 2018 Summer School on Computational Interaction, University of Cambridge, (JHW, AO, POK)

- 2019 ACM SIGCHI Tutorial on Bayesian Interaction, Glasgow, United Kingdom, (AO, JHW, POK)

- 2019Summer School on Computational Interaction, Columbia University, (AO)

All of the prior events had between 15 and 30 attendees.

\section{PARTICIPANTS}

We would expect 15-25 participants to attend for this tutorial. We would expect to cap participation at a maximum of 25 participants to ensure that the practical material can realistically be delivered in time available. We would expect the primary audience for this tutorial to be $\mathrm{PhD}$ students and postdocs involved in HCI research, but we welcome other academics and industry practitioners.

\section{FORMAT}

This tutorial will be a full-day event. The tutorial day will consist of interwoven lectures and small practical exercises, with live coding and experimentation. This format will give attendees time to absorb concepts and apply them to HCI problems with the support of the presenters. We have successfully applied this alternating lecture/practical format in prior tutorials.

\subsection{Schedule}

Each of the morning and afternoon sessions will comprise approximately 2.5 hours of lectures delivered over a four hour period. There will be extensive breaks for discussion and hands-on practical break-out sessions.

\section{- Morning}

- Introduction to Bayesian approaches in HCI: why Bayesian?

- Practical bayesian optimisation for interaction design.

\section{- Afternoon}

- Probabilistic inference for discrete symbols: Bayesian approaches to text entry
- Sequential Monte Carlo for online inference of user intention.

\subsection{Technologies and format of notes}

We will make extensive use of Jupyter notebooks to provide interactive notes that combine executable Python examples with inline explanations/lecture notes. This is an approach we have very successfully applied over the last five years. We will use the MyBinder ${ }^{1}$ service to provide immediate access to the materials without any user-side install, as well as downloadable versions of our materials.

\section{OUTCOMES}

- Philosophy We will have introduced students to Bayesian approaches to thinking about interaction design, and the fundamental change of perspective this implies. Students will have a clear understanding of the role of uncertainty, and the distinction between considering distributions over parameters and point estimates.

- Theory Participants will be familiar with the ideas of Bayesian inference, including stochastic approximation approaches for online updates (MCMC/sequential Monte Carlo), nonparametric inference (Gaussian processes) and their relationship to traditional techniques and algorithms.

- Application Participants will know how to identify applications of Bayesian methods in human-computer interaction at both design-time and run-time and apply these computational techniques in interface designs that correctly propagate and update uncertainty.

- Practical Participants will have used standard Python libraries and code samples to practically implement and experiment with these techniques and had an opportunity for in-depth exploration of the implementation of Bayesian methods in interaction design.

- Output We will publish the full, executable notes for the tutorial online, as we have for previous tutorial sessions. 